\begin{tabular}{|l|l|l|l|l|l|l|}
\hline InterteXto & Uberaba & UFTM & $\begin{array}{l}\text { v. 2 } \\
\text { n. 2 }\end{array}$ & p. 20-38 & 2009 - jul. / dez. & ISSN 1981-0601 \\
\hline
\end{tabular}

\title{
UMA ANÁLISE SEMIÓTICA DO POEMA ANNABEL LEE, DE EDGAR ALLAN POE
}

\author{
A SEMIOTIC ANALYSIS OF THE POEM ANNABEL LEE, BY EDGAR ALLAN \\ POE
}

Fernanda Cristina Araújo Batista ${ }^{1}$

Resumo: Este trabalho visa a realizar uma análise do poema Annabel Lee, de Edgar Allan Poe, sob as perspectivas da semiótica greimasiana. Utilizamos a teoria do percurso gerativo de sentido, iniciando a análise pelas estruturas do nível narrativo para passar àquelas do nível discursivo e, por fim, chegar às estruturas do nível fundamental. Também fizemos referências ao romance Lolita, de Vladimir Nabokov, que retoma o poema, a fim de compreender como e por que ocorreu essa retomada.

Palavras-chave: Semiótica greimasiana; percurso gerativo de sentido; Annabel Lee.

Abstract: This paper aims analyzing the poem Annabel Lee, by Edgar Allan Poe, under the perspectives of Greimasian semiotics. We used the theory of the generative trajectory of meaning and started the study with an analysis of its surface semionarrative structures in order to reach its discursive structures, finally getting to its deep semio-narrative structures. We also mentioned Vladimir Nabokov's novel Lolita, which refers to the poem, in order to understand how and why this reference was made.

Keywords: Greimasian semiotics; generative trajectory of meaning; Annabel Lee.

\section{Introdução}

Annabel Lee, escrito por Edgar Allan Poe em 1849, é um poema de seis estrofes que consiste na descrição das causas, significados e conseqüências da morte da menina que dá título à obra.

Realizaremos este trabalho, segundo a semiótica greimasiana, à luz da teoria do percurso gerativo de sentido: partiremos do nível narrativo em direção ao nível discursivo, chegando, por fim, ao nível fundamental. Para iniciarmos a análise do nível narrativo, dividiremos o poema em seis seqüências. Já para

\footnotetext{
${ }^{1}$ Universidade Presbiteriana Mackenzie. fcabatista@hotmail.com
} 


\begin{tabular}{|l|l|l|l|l|l|l|}
\hline InterteXto & Uberaba & UFTM & $\begin{array}{l}\text { v. 2 } \\
\text { n. } 2\end{array}$ & p. 20-38 & 2009 - jul. / dez. & ISSN 1981-0601 \\
\hline
\end{tabular}

realizarmos as análises discursiva e fundamental, usaremos o poema como um todo.

Trataremos, também, da questão da intertextualidade presente no romance Lolita, de Vladimir Nabokov, a fim de verificar de que forma o poema de Poe é recontextualizado por Nabokov mais de cem anos depois de sua publicação.

\section{Nível narrativo}

Para realizarmos a análise do nível narrativo do poema Annabel Lee, o dividiremos em seis seqüências, nas quais se apresentam ora enunciados de estado, aqueles em que aparece uma relação de junção entre um sujeito e um objeto, ora enunciados de fazer, aqueles em que é relatada uma transformação de um enunciado de estado em outro (BARROS, 2000, p. 18, 19).

Na primeira seqüência, é relatado pelo actante $S 1$, enquanto sujeito da enunciação, um enunciado de estado em que o actante S2, Annabel Lee, é um sujeito virtualizado, isto é, possui um querer-ser com relação à conjunção com um objeto de valor desejável, o qual é o próprio S1, porém, enquanto sujeito da narrativa, a quem ela quer amar e por quem deseja ser amada:

\footnotetext{
It was many and many a year ago,

In a kingdom by the sea,

That a maiden there lived whom you may know

By the name of ANNABEL LEE;

And this maiden she lived with no other thought

Than to love and be loved by me.
}

S2 quer $\cap$ Ov (S1)

Esse amor não aparece como um amor concreto, mas sim como uma espera por parte de S2, conforme se pode ler nos versos "And this maiden she lived with no other thought/ Than to Love and be loved by me" e é o momento em que S1 (sujeito) adquire a competência do poder-ser, pois, se S2 possui o quer-ser amada por ele, ele passa a possuir o poder-ser com relação a ela. 


\begin{tabular}{|l|l|l|l|l|l|l|}
\hline InterteXto & Uberaba & UFTM & $\begin{array}{l}\text { v. 2 } \\
\text { n. } 2\end{array}$ & p. 20-38 & 2009 - jul. / dez. & ISSN 1981-0601 \\
\hline
\end{tabular}

$\mathrm{Na}$ segunda seqüência, por sua vez, aparecem três enunciados de estado.

Dois deles dizem respeito a $\mathrm{S} 1$ (sujeito) e S2: $\mathrm{S} 1$ (sujeito) também apresenta um querer-ser com relação a $S 2$ e ambos já estão em conjunção com seu Ov desejável, sendo, assim, sujeitos atualizados e realizados, o que pressupõe que podem-ser amantes e de fato o são, ainda que sejam duas crianças. Ademais, querendo e crendo que poderão continuar em conjunção um com o outro e, conseqüentemente, com sua relação amorosa, tanto $\mathrm{S} 1$ (sujeito) quanto S2 se encontram numa espera confiante, isto é, num estado de alegria:

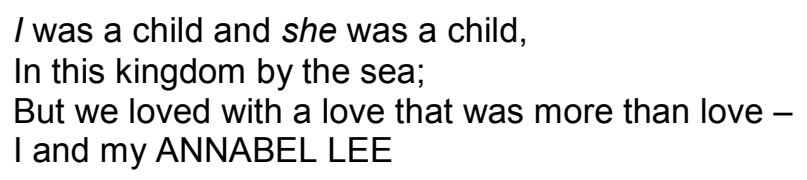

$S 1 \cap \operatorname{Ov}(S 2)$

\section{S2 $\cap$ Ov (S1)}

No terceiro enunciado, segundo conta o narrador, aparece o anti-sujeito S3, que são os serafins do paraíso, e é também um sujeito virtualizado por possuir o querer-ser com relação ao amor de S1 (sujeito) e S2:

With a love that the winged seraphs of heaven

Coveted her and me

\section{S3 $\cap$ Ov (amor de S1 e S2)}

Segundo o narrador, os serafins invejam S1 (sujeito) e S2 porque o amor entre eles é demasiadamente grande, unindo corpo e alma, algo que aqueles não podem obter por serem as entidades celestes responsáveis pela transmissão do amor, e não pela sua vivência (a qual é peculiar aos seres humanos).

Em Semiótica das Paixões: dos estados de coisas aos estados de alma (1993, p. 176), Greimas e Fontanille descrevem um tipo de inveja como "um sentimento de tristeza, de irritação ou de ódio que nos anima contra quem possui um bem que não temos". Assim, essa inveja que sente S3 leva a um 


\begin{tabular}{|l|l|l|l|l|l|l|}
\hline InterteXto & Uberaba & UFTM & $\begin{array}{l}\text { v. 2 } \\
\text { n. 2 }\end{array}$ & p. 20-38 & 2009 - jul. / dez. & ISSN 1981-0601 \\
\hline
\end{tabular}

programa narrativo de privação por espoliação nas terceira e quarta seqüências: S3 realiza uma ação malevolente com relação a S1 (sujeito) e S2 numa tentativa de acabar com esse sentimento que lhe causa tristeza, irritação ou ódio por meio do aniquilamento de S2.

Por sua alta posição celestial, isto é, por pertencer a mais alta hierarquia dos anjos, S3 é também um sujeito potencializado e atualizado, isto é, detém o saber e o poder-fazer o que almeja. Assim sendo, consegue fazer com que S2 adoeça ao enviar um vento através das nuvens:

And this was the reason that, long ago,

In this kingdom by the sea,

A wind blew out of a cloud, chilling

My beautiful ANNABEL LEE

S3 (serafins) $\rightarrow[S 2$ (Annabel) $\cap$ Ov (saúde) $\rightarrow S 2$ (Annabel) U Ov (saúde)]

Essa ação de S3 leva S1 (sujeito), que estava numa espera alegre e confiante por crer poder manter a conjunção em que estava, a uma suspensão dessa alegria e confiança:

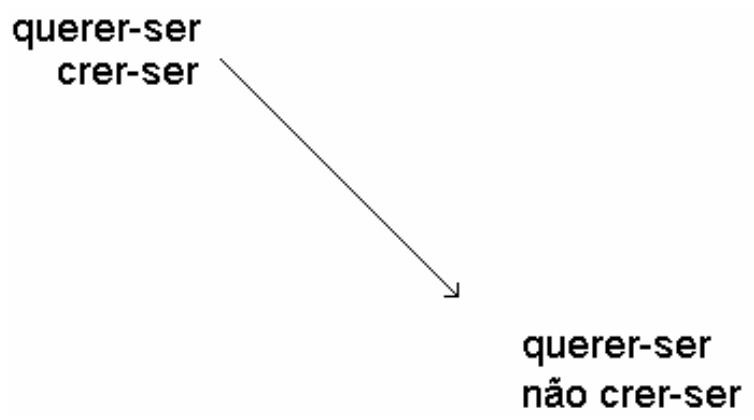

Na quarta seqüência, S3 torna-se sujeito realizado ao acabar matando S2, assim colocando-a em disjunção com a vida e com a relação amorosa com S1 (sujeito). Aqui, porém, aparece outro anti-sujeito, S4 (familiares de S2), que coloca S1 (sujeito) em disjunção com o corpo morto de S2 ao levá-lo para longe dele com o propósito de que seja enterrado:

So that her highborn kinsmen came

And bore her away from me,

To shut her up in a sepulchre

In this kingdom by the sea. 


\begin{tabular}{|l|l|l|l|l|l|l|}
\hline InterteXto & Uberaba & UFTM & $\begin{array}{l}\text { v. 2 } \\
\text { n. } 2\end{array}$ & p. 20-38 & 2009 - jul. / dez. & ISSN 1981-0601 \\
\hline
\end{tabular}

The angels, not half so happy in heaven,

Went envying her and me -

Yes! - that was the reason (as all men know,

In this kingdom by the sea)

That the wind came out of the cloud by night,

Chilling and killing my ANNABEL LEE.

S3 (serafins) $\rightarrow$ [S2 (Annabel) $\cap$ Ov (vida) $\rightarrow$ S2 U Ov (vida)]

S3 (serafins) $\rightarrow[S 2$ (Annabel) $\cap$ Ov (relação de amor com S1) $\rightarrow$ S2 U Ov (relação de amor com S1)]

$S 4$ (familiares de S2) $\rightarrow[S 1$ (narrador) $\cap$ Ov (corpo de S2) $\rightarrow S 1 \cup$ Ov (corpo de S2)]

Assim, leva-se S1 (sujeito) à amargura por ter perdido seu objeto de valor:

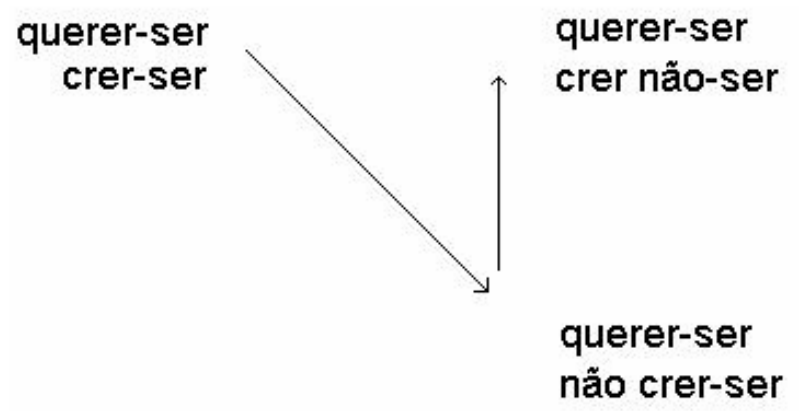

$\mathrm{Na}$ quinta seqüência, esse fazer de S3 leva S1 (sujeito), que se encontrava num estado de amargura por saber que não poderá obter mais a conjunção com o amor deste em forma de relação de corpo e alma, a retornar para o eixo da alegria e satisfação, pois este afirma que seu amor por S2 era tão grande que nada, nem a morte, pode acabar com ele, permanecendo, assim, o sentimento, ainda que a manifestação física tenha cessado:

\footnotetext{
But our love it was stronger by far than the love

Of those who were older than we -

Of many far wiser than we -

And neither the angels in heaven above,

Nor the demons down under the sea,

Can ever dissever my soul from the soul

Of the beautiful ANNABEL LEE.
} 


\begin{tabular}{|l|l|l|l|l|l|l|}
\hline InterteXto & Uberaba & UFTM & $\begin{array}{l}\text { v. 2 } \\
\text { n. } 2\end{array}$ & p. 20-38 & 2009 - jul. / dez. & ISSN 1981-0601 \\
\hline
\end{tabular}

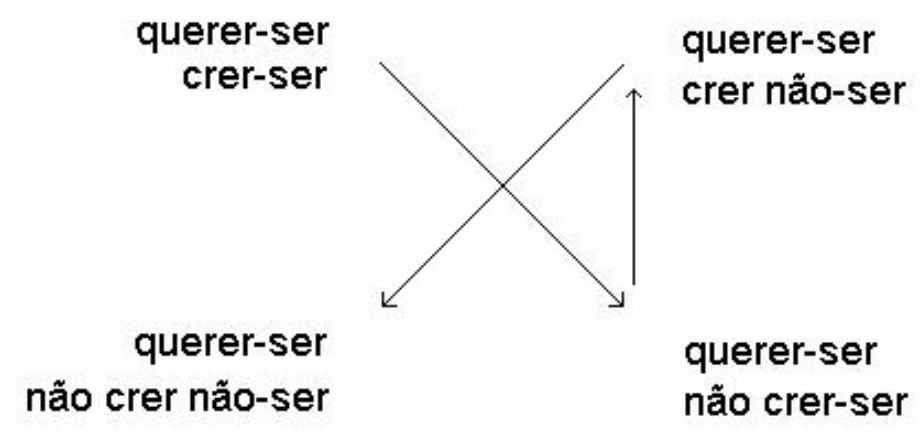

Na sexta e última seqüência, temos então que S1 aparece como sujeito da enunciação, e não mais como sujeito da narrativa, porém, aparece para dar continuidade a esta, voltando ao querer e crer-ser, pois se coloca em conjunção com Annabel.

Dessa forma, sabemos que, a princípio, S1 (sujeito) desejava e mantinha uma relação corpo e alma com S2, e que isso passa a não ser mais possível, sendo tangível apenas a continuidade do amor de S1 (sujeito) à memória de S2 e ao túmulo onde esta está enterrada, fato que não modifica seu amor, pois ele aceita essa nova condição e assim pode manter a conjunção, não mais de corpo e alma, mas apenas de alma.

\footnotetext{
For the moon never beams without bringing me dreams

Of the beautiful ANNABEL LEE;

And the stars never rise but I feel the bright eyes

Of the beautiful ANNABEL LEE;

And so, all the night-tide, I lie down by the side

Of my darling- my darling- my life and my bride,

In the sepulchre there by the sea, -

In her tomb by the sounding sea.
}

S1 ก Ov (amor à memória de S2)

querer-ser

crer-ser

querer-ser

não crer não-ser querer-ser

crer não-ser

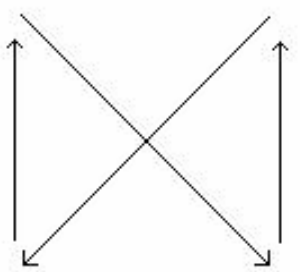

querer-ser

não crer-ser 


\begin{tabular}{|l|l|l|l|l|l|l|}
\hline InterteXto & Uberaba & UFTM & $\begin{array}{l}\text { v. 2 } \\
\text { n. } 2\end{array}$ & p. 20-38 & 2009 - jul. / dez. & ISSN 1981-0601 \\
\hline
\end{tabular}

Temos, então, que a paixão se S1 é um apego intenso à junção com S2. Segundo Greimas e Fontanille (1993, p. 182, 184), o apego intenso é considerado uma necessidade de junção do sujeito com o objeto que tem o investimento total daquele, que não existiria sem ela. Assim, para o sujeito, esse apego não sofre as variações efetivas que ocorrem na relação entre ele e o objeto, isto é, mesmo que seu objeto tenha desaparecido ou morrido, o sujeito pode continuar a sonhar que é conjunto com ele. Daí o narrador resistir à perda de Annabel e continuar a sentir-se conjunto com ela mesmo após a sua morte.

\section{Nível discursivo}

\section{Sintaxe do discurso}

Neste nível, trataremos do modo como o poema é aspectualizado, isto é, analisaremos as maneiras como são definidas as questões de actorialização, temporalização e espacialização dentro do texto.

Para tratar das pessoas que aparecem em Annabel Lee, levamos em consideração que este poema foi construído visando a produzir um efeito de proximidade do sujeito da enunciação, que se utilizou, assim, da desembreagem enunciativa, isto é, narrou-o em primeira pessoa, técnica usada para esse fim (BARROS, 2000, p. 55).

No poema, aparecem seis atores: um eu narrador de primeira pessoa; um eu sujeito da narrativa; um ele Annabel Lee; um nós exclusivo: eu (narrador) e ele (Annabel Lee); um eles serafins e um eles familiares de Annabel.

O ele Annabel aparece na primeira estrofe quando o narrador diz que há muito tempo, num reino à beira mar, vivia uma moça virgem: That a maiden there lived whom you may know/ By the name of Annabel Lee. Instala-se, aí, 


\begin{tabular}{|l|l|l|l|l|l|l|}
\hline InterteXto & Uberaba & UFTM & $\begin{array}{l}\text { v. 2 } \\
\text { n. } 2\end{array}$ & p. 20-38 & 2009 - jul. / dez. & ISSN 1981-0601 \\
\hline
\end{tabular}

um enunciatário você, que possivelmente conheça esse ele de quem se fala, o qual desejava amar e ser amada por esse eu que continua narrando: And this maiden she lived with no other thought/ Than to love and be loved by me (grifos nossos).

O nós exclusivo aparece no terceiro verso da segunda estrofe, quando o narrador conta que ele, quando criança, vivia uma relação amorosa com o ele Annabel: But we loved with a love that was more than love-/l and my Annabel Lee (grifo nosso).

O eles serafins aparece no quinto verso da segunda estrofe quando se diz que eles tinham inveja daquele nós narrador e Annabel: (...) a love that the winged seraphs of heaven/Coveted her and me (grifo nosso).

Finalmente, o ele que são os familiares de Annabel aparece no quinto verso da terceira estrofe ao cumprir o papel de levar o corpo de Annabel para ser enterrado: (...) that her highborn kinsmen came/ And bore her away from me,/To shut her up in a sepulchre.

Reaparece, assim, o nós exclusivo quando o narrador reafirma que os anjos invejavam os dois, e que o amor de ambos era mais forte do que o amor de adultos: The angels, not half so happy in heaven,/ Went envying her and me-(...) But our love it was stronger by far than the love/ Of those who were older than we-/ Of many far wiser than we (grifos nossos).

Ressurge, também, o eu, mas agora como sujeito da enunciação, que contém em si o sujeito da narrativa, bem como o ele Annabel, com quem outrora havia estado conjunto, e de quem, depois, foi disjunto. Percebemos isso quando o enunciador, apesar de Annabel ter morrido e sido levada para longe dele, chama-a de amor, vida, noiva, e diz que sonha com ela, sente seus olhos toda noite e que se deita ao lado do túmulo onde ela está enterrada: For the moon never beams without bringing me dreams (...) And the stars never rise but I feel the bright eyes (...) And so, all the night-tide, I lie down by the side/ Of my darling- my darling- my life and my bride (grifos nossos). 


\begin{tabular}{|l|l|l|l|l|l|l|}
\hline InterteXto & Uberaba & UFTM & $\begin{array}{l}\text { v. 2 } \\
\text { n. 2 }\end{array}$ & p. 20-38 & 2009 - jul. / dez. & ISSN 1981-0601 \\
\hline
\end{tabular}

O efeito que causa a presença dessas pessoas no discurso é o de ter havido um eu (sujeito da narrativa) que se juntou a um ele (Annabel Lee) e formou um nós feliz (eu e Annabel Lee), o qual um eles (serafins) separou fisicamente por meio de um ele (familiares que a enterraram), mas não conseguiu distanciar totalmente, pois que o eu já tinha contido em si a junção com esse ele, que permaneceu mesmo após a morte e a passagem do tempo. Quem nos conta isso é um eu adulto (sujeito da enunciação) que ainda vê e sente como o eu sujeito, que era uma criança quando participou da narrativa, utilizando-se, para isso, de um misto de linguagem adulta e linguagem infantil.

Para tratar da aspectualização temporal, faremos uma tradução livre do poema além de nos referirmos a ele diretamente.

O tempo de referência do texto é um tempo também enunciativo, construído pelo emprego de um presente durativo, o simple present tense do inglês, e está ligado à idéia de as pessoas ele e eu, agora, estarem fundidos num nós inseparável ainda que um deles tenha morrido. No entanto, narra-se, de forma predominante no poema, o que acontecia, também durativamente, antes desse presente, ou seja, num tempo enuncivo, até que ocorreu um acontecimento pontual que modificou toda a narrativa para o modo como ela se encontra nesse momento do agora.

Nas duas primeiras estrofes, usa-se o simple past tense do inglês, que, aparecendo junto à marcação temporal "many and many a year ago" (muitos e muitos anos atrás), no primeiro verso, causa o efeito de que o narrado ocorria extensivamente num passado longínquo: Annabel Lee vivia com o único pensamento de ser amada pelo narrador; ambos eram crianças, e se amavam com um amor tão grande que os serafins o invejavam:

(...) a maiden there lived (...)

And this maiden she lived with no other thought (...)

I was a child and she was a child (...)

But we loved with a love that was more than love - (...)

With a love that the winged seraphs in Heaven

Coveted her and me. (grifos nossos) 


\begin{tabular}{|l|l|l|l|l|l|l|}
\hline InterteXto & Uberaba & UFTM & $\begin{array}{l}\text { v. 2 } \\
\text { n. } 2\end{array}$ & p. 20-38 & 2009 - jul. / dez. & ISSN 1981-0601 \\
\hline
\end{tabular}

Nas terceira e quarta estrofes, continua a ser usado o simple past tense, mas agora com o aspecto de pontualidade, intenso: um vento soprou das nuvens, os familiares de Annabel vieram e levaram-na para longe do narrador:

\author{
A wind blew out of a cloud (...) \\ So that her high-born kinsmen came \\ And bore her away from me
}

Mesmo quando se usam os verbos chill e kill no gerúndio, trata-se de uma opção estilística usada com a finalidade de reduzir orações coordenadas aditivas assindética e sindética, respectivamente, cujos verbos estariam também no simple past tense caso não houvessem sido reduzidas: um vento soprou das nuvens, causando calafrios em Annabel e matando-a seria um vento soprou das nuvens, causou calafrios em Annabel e matou-a:

\title{
(...) the wind came out of the cloud by night, Chilling and killing my ANNABEL LEE.
}

Nos primeiro e segundo versos da quinta estrofe, modifica-se novamente o tempo para o simple past tense com o aspecto durativo - o amor de Annabel e do narrador era mais forte que aquele que sentiam as pessoas mais velhas e, no sexto verso da mesma estrofe, passa-se a usar o simple present tense do inglês com o aspecto extenso, que dura, intensificado pelo uso do verbo modal can junto do advérbio de tempo ever: ninguém pode jamais separar as almas do narrador e de Annabel Lee; a lua nunca brilha sem que ele sonhe com ela; as estrelas nunca sobem ao céu sem que ele sinta os olhos brilhantes da menina; durante toda a noite ele se deita ao lado do túmulo de sua amada:

But our love it was stronger by far than the love

Of those who were older than we (...)

And neither the angels in Heaven above,

Nor the demons down under the sea,

Can ever dissever my soul from the soul

Of the beautiful ANNABEL LEE: -

For the moon never beams, without bringing me dreams

Of the beautiful ANNABEL LEE;

And the stars never rise, but I feel the bright eyes

Of the beautiful Annabel Lee:

And so, all the night-tide, I lie down by the side

Of my darling — my darling — my life and my bride (...) 


\begin{tabular}{|l|l|l|l|l|l|l|}
\hline InterteXto & Uberaba & UFTM & $\begin{array}{l}\text { v. 2 } \\
\text { n. 2 }\end{array}$ & p. 20-38 & 2009 - jul. / dez. & ISSN 1981-0601 \\
\hline
\end{tabular}

O espaço de referência é aquele onde o eu narrador está agora: junto de sua amada, deitado ao lado de seu túmulo, próximo ao barulho do mar. No entanto, percebe-se que esse espaço não é pragmático, e sim cognitivo, pois usa-se um lá para falar dele: And so, all the night-tide, I lie down by the side/ Of my darling- my darling- my life and my bride,/ In the sepulchre there by the sea,/ In her tomb by the sounding sea. Obtemos, assim, a idéia de que o narrador não está realmente ao lado dela, mas, sim, em pensamento.

O espaço anterior, onde o eu sujeito da narrativa vivia com o ele, era também um reino à beira mar, como demonstra o segundo verso da primeira, da segunda e da terceira estrofes: In a kingdom by the sea. Temos, então, que, neste espaço, tanto Annabel quanto o enunciador estavam presentes, já que foi nele que a narrativa se passou.

Essa utilização de um espaço do aqui em contraposição a um espaço do lá gera o efeito de distanciamento daquele e de proximidade deste, o que completa a idéia do tempo passado, acabado, em que o amor foi vivenciado em sua manifestação física e espiritual, e do tempo presente, que dura, em que o amor é vivenciado em sua forma espiritual apenas.

\section{Semântica do discurso}

As figuras recorrentes no poema Annabel Lee podem ser divididas em dois tipos.

Há figuras que indicam o tema do amor idealizado. São: kingdom by the sea, maiden, thought, child, heaven, moon, stars, bright eyes, darling, life, bride, beautiful, iconizadas por Annabel Lee.

Há figuras que indicam o tema da morte que vem interromper a vida e o amor, mas que, neste texto, fracassa. São: wind, cloud, sepulchre, demons, tomb e são iconizadas pelos seraphs e angels.

A presença dessas figuras indica o tema maior de que trata o poema: amor e morte, com aquele durando mesmo após a morte de um dos amantes, 


\begin{tabular}{|l|l|l|l|l|l|l|}
\hline InterteXto & Uberaba & UFTM & $\begin{array}{l}\text { v. 2 } \\
\text { n. } 2\end{array}$ & p. 20-38 & 2009 - jul. / dez. & ISSN 1981-0601 \\
\hline
\end{tabular}

o que demonstra uma perspectiva cristã sob a qual foi narrado o misticismo da vida após a morte e o romantismo desse amor que não deixa de existir nem mesmo quando um dos amantes desaparece.

\section{Nível fundamental}

Podemos dizer que a oposição fundamental do texto é vida $X$ morte devido aos programas narrativos que aparecem no nível narrativo do poema bem como às figuras e temas de seu nível discursivo.

A vida é representada de maneira eufórica e a morte disfórica, e o quadrado semiótico, que se inicia na vida, passa pela não-vida, pela morte, pela não-morte e retorna, enfim, à vida.

A vida aparece no estado inicial, ou seja, quando se dá a conjunção do narrador com Annabel Lee, logo no início do poema:

I was a child and she was a child,

In this kingdom by the sea;

But we loved with a love that was more than love -

I and my ANNABEL LEE

A não-vida é disfórica e é caracterizada pelo momento em que Annabel adoece:

\section{A wind blew out of a cloud, chilling \\ My beautiful ANNABEL LEE;}

\section{vida}

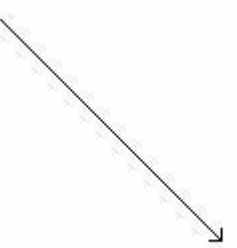

\section{não-vida}

A morte, por sua vez, aparece quando Annabel Lee falece e é levada para longe do narrador:

So that her high-born kinsmen came

And bore her away from me,

To shut her up in a sepulcher 


\begin{tabular}{|l|l|l|l|l|l|l|}
\hline InterteXto & Uberaba & UFTM & $\begin{array}{l}\text { v. 2 } \\
\text { n. 2 }\end{array}$ & p. 20-38 & 2009 - jul. / dez. & ISSN 1981-0601 \\
\hline
\end{tabular}

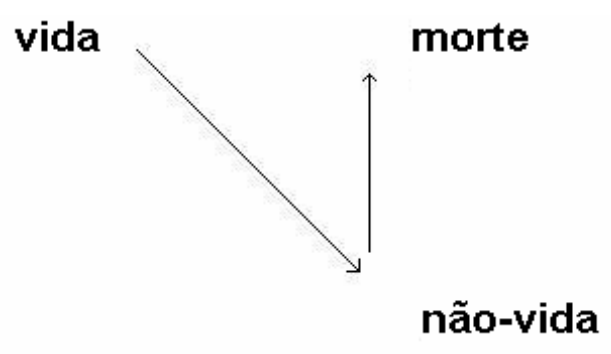

Chega-se à não-morte quando o narrador afirma que o amor vivido com Annabel é maior do que o poder de qualquer anjo ou demônio e que, por isso, nada poderá separar suas almas, isto é, há o retorno a um estado eufórico quando se afirma a supremacia do apego sobre a morte:

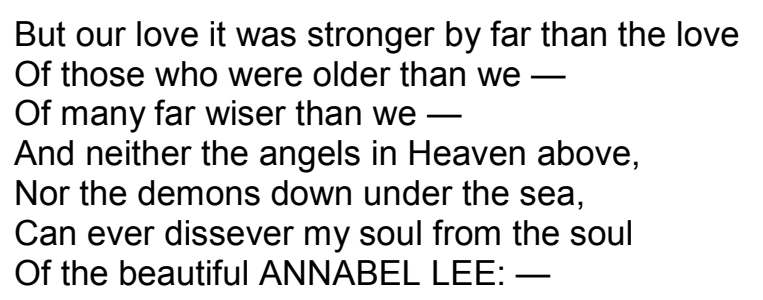

vida

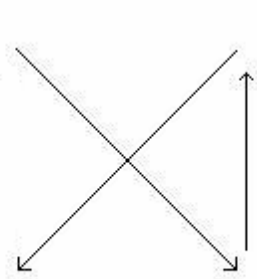

não-morte morte

\section{não-vida}

Acreditando permanecer ao lado de Annabel mesmo que isso signifique, em pensamento, passar as noites junto a seu túmulo, o narrador volta ao estado eufórico de vida, pois revê seu objeto de valor:

For the moon never beams, without bringing me dreams

Of the beautiful ANNABEL LEE;

And the stars never rise, but I feel the bright eyes

Of the beautiful Annabel Lee:

And so, all the night-tide, I lie down by the side

Of my darling - my darling - my life and my bride,

In her sepulchre there by the sea -

In her tomb by the sounding sea. 


\begin{tabular}{|l|l|l|l|l|l|l|}
\hline InterteXto & Uberaba & UFTM & $\begin{array}{l}\text { v. 2 } \\
\text { n. } 2\end{array}$ & p. 20-38 & 2009 - jul. / dez. & ISSN 1981-0601 \\
\hline
\end{tabular}

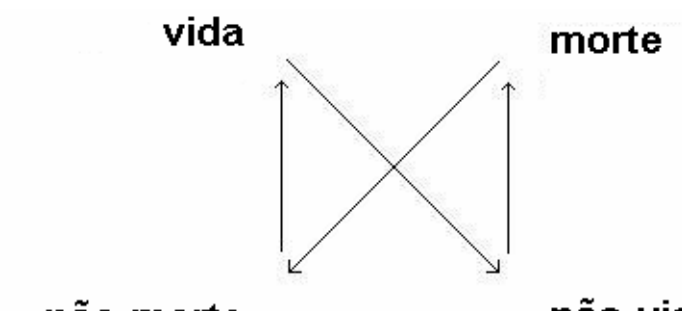

não-morte não-vida

\section{Intertextualidade}

Segundo José Luiz Fiorin (BARROS e FIORIN, 2003, p. 30), “a intertextualidade é o processo de incorporação de um texto em outro, seja para reproduzir o sentido incorporado, seja para transformá-lo". Considerando essa definição, pode-se dizer que o romance Lolita (1955), de Vladimir Nabokov, que foi publicado mais de cem anos após o poema Annabel Lee, de Poe, já nas primeiras páginas demonstra intertextualidade em relação a este por meio do processo de citação de elementos semânticos do poema. Analisaremos algumas das citações mais importantes que o romance faz do poema de Allan Poe justificando o seu emprego.

Já no primeiro capítulo do romance, o narrador Humbert Humbert cita o poema quando começa por explicar quem foi a personagem título de seu livro e diz que não teria existido uma Lolita se, anos antes, ele não houvesse amado outra menina num "princedom by the sea":

Did she have a precursor? She did, indeed she did. In point of fact, there might have been no Lolita at all had I not loved, one summer, an initial girl-child. In a princedom by the sea. (NABOKOV, 1999, p. 3)

Ele ainda completa:

Ladies and gentlemen of the jury, exhibit number one is what the seraphs, the misinformed, simple, noble-winged seraphs, envied. Look at this tangle of thorns. (NABOKOV, 1999, p. 3)

Ao mencionar algo de que os serafins sentiam inveja, faz uma citação de um percurso figurativo do poema, pois neste aparece a inveja dos serafins como causa da morte de Annabel. 


\begin{tabular}{|l|l|l|l|l|l|l|}
\hline InterteXto & Uberaba & UFTM & $\begin{array}{l}\text { v. } 2 \\
\text { n. } 2\end{array}$ & p. 20-38 & 2009 - jul. / dez. & ISSN 1981 -0601 \\
\hline
\end{tabular}

O narrador diz que, aos treze anos, conheceu Annabel Leigh, menina de sobrenome de escrita diferente daquele que tem a menina do poema, mas cuja pronúncia, em inglês, é exatamente a mesma. Ele afirma terem se apaixonado intensamente, mas não terem podido concretizar esse amor por conta da vigilância das famílias:

All at once we were madly, clumsily, shamelessly, agonizingly in love with each other; hopelessly, I should add, because that frenzy of mutual possession might have been assuaged only by our actually imbibing and assimilating every particle of each other's soul and flesh; but there we were, unable even to mate as slum children would have so easily found an opportunity to do so. (NABOKOV, 1999, p. 5, 6)

No trecho seguinte, acrescenta à sua narrativa uma nuance sexual que não aparecia claramente no poema:

I have reserved for the conclusion of my "Annabel" phase the account of our unsuccessful first tryst. One night, she managed to deceive the vicious vigilance of her family. In a nervous and slender-leaved mimosa grove at the back of their villa we found a perch on the ruins of a low stone wall. Through the darkness and the tender trees we could see the arabesques of lighted windows which, touched up by the colored inks of sensitive memory, appear to me now like playing cards-presumably because a bridge game was keeping the enemy busy. She trembled and twitched as I kissed the corner of her parted lips and the hot lobe of her ear. A cluster of stars palely glowed above us, between the silhouettes of long thin leaves; that vibrant sky seemed as naked as she was under her light frock. I saw her face in the sky, strangely distinct as if it emitted a faint radiance of its own. Her legs, her lovely live legs, were not too close together, and when my hand located what it sought, a dreamy and eerie expression, half pleasure, half-pain, came over those childish features. She sat a little higher than I, and whenever in her solitary ecstasy she was led to kiss me, her head would bend with a sleepy, soft, drooping movement that was almost woeful, and her bare knees caught and compressed my wrist, and slackened again and her quivering mouth, distorted by the acridity of some mysterious potion, with a sibilant intake of breath came near to my face. She would try to relieve the pain of love by first roughly rubbing her dry lips against mine; then my darling would draw away with a nervous toss of her hair, and then again come darkly near and let me feed on her open mouth, while with a generosity that was ready to offer her everything, my heart, my throat, my entrails, I gave her to hold in her awkward fist the scepter of my passion. (NABOKOV, 1999, p. 7)

Esse acréscimo serve a um propósito do narrador de Lolita, que é convencer o leitor que a patologia de que sofre em sua vida adulta, a pedofilia, iniciou-se com a frustração sofrida, na infância, pela privação da possibilidade 


\begin{tabular}{|l|l|l|l|l|l|l|}
\hline InterteXto & Uberaba & UFTM & $\begin{array}{l}\text { v. 2 } \\
\text { n. } 2\end{array}$ & p. 20-38 & 2009 - jul. / dez. & ISSN 1981-0601 \\
\hline
\end{tabular}

de ter vivido um amor de corpo e alma com Annabel Leigh. Essa informação serve para que ele se questione acerca da causa dessa sua condição, escrevendo no quarto capítulo:

I leaf again and again through these miserable memories, and keep asking myself, was it then, in the glitter of that remote summer, that the rift in my life began; or was my excessive desire for that child only the first evidence of an inherent singularity? When I try to analyze my own cravings, motives, actions and so forth, I surrender to a sort of retrospective imagination which feeds the analytic faculty with boundless alternatives and which causes each visualized route to fork and re-fork without end in the maddeningly complex prospect of my past. I am convinced, however, that in a certain magic and fateful way Lolita began with Annabel.

I also know that the shock of Annabel's death consolidated the frustration of that nightmare summer, made of it a permanent obstacle to any further romance throughout the cold years of my youth. The spiritual and the physical had been blended in us with a perfection that must remain incomprehensible to the matter-of-fact, crude, standardbrained youngsters of today. Long after her death I felt her thoughts floating through mine. Long before we met we had had the same dreams. We compared notes. We found strange affinities. The same June of the same year (1919) a stray canary had fluttered into her house and mine, in two widely separated countries. Oh, Lolita, had you loved me thus! (...)

But that mimosa grove--the haze of stars, the tingle, the flame, the honey-dew, and the ache remained with me, and that little girl with her seaside limbs and ardent tongue haunted me ever since--until at last, twenty-four years later, I broke her spell by incarnating her in another. (NABOKOV, 1999, p. 6, 8)

Temos, então, que a escolha de Nabokov em fazer de Humbert um pedófilo que afirma sê-lo como conseqüência de um trauma de infância é uma homenagem ao poema de Poe, sendo um "final" para a história de seu narrador.

No entanto, como se segue no romance, a citação do poema é, além de um possível final para a narrativa deste, uma forma de descrever a menina Lolita diferentemente da menina que fora Annabel e o narrador do romance, também diferentemente do que fora o narrador do poema. Nabokov, em seu romance, desfaz a imagem romântica de Annabel em Lolita e desfaz a apatia também romântica do narrador do poema em Humbert: ela não é mais uma moça virgem e frágil que desejava intensamente amar o narrador e ele 


\begin{tabular}{|l|l|l|l|l|l|l|}
\hline InterteXto & Uberaba & UFTM & $\begin{array}{l}\text { v. 2 } \\
\text { n. } 2\end{array}$ & p. 20-38 & 2009 - jul. / dez. & ISSN 1981-0601 \\
\hline
\end{tabular}

tampouco permanece inabalável ao lado de seu túmulo, mas leva uma vida longe do reino (ou principado!) à beira-mar, ainda que continue projetando-o em outros espaços:

\begin{abstract}
Despite my having dabbled in psychiatry and social work, I really knew very little about children. After all, Lolita was only twelve, and no matter what concessions I made to time and place--even bearing in mind the crude behavior of American schoolchildren--I still was under the impression that whatever went on among those brash brats, went on at a later age, and in a different environment. Therefore (to retrieve the thread of this explanation) the moralist in me by-passed the issue by clinging to conventional notions of what twelve-year-old girls should be. The child therapist in me (a fake, as most of them are--but no matter) regurgitated neo-Freudian hash and conjured up a dreaming and exaggerating Dolly in the "latency" period of girlhood. Finally, the sensualist in me (a great and insane monster) had no objection to some depravity in his prey. But somewhere behind the raging bliss, bewildered shadows conferred--and not to have heeded them, this is what I regret! Human beings, attend! I should have understood that Lolita had already proved to be something quite different from innocent Annabel, and that the nymphean evil breathing through every pore of the fey child that I had prepared for my secret delectation, would make the secrecy impossible, and the delectation lethal. I should have known (by the signs made to me by something in Lolita-the real child Lolita or some haggard angel behind her back) that nothing but pain and horror would result from the expected rapture. (NABOKOV, 1999, p. 89)
\end{abstract}

Temos, dessa forma, que Nabokov, ao construir Humbert, que, por sua vez, constrói Lolita de acordo com olhos que viram e amaram a Annabel de Poe, mas depois descobre que o que vê não é o real, mostra não só o mundo de toda a literatura romântica, mas também o mundo dos leitores desse tipo de obra, de forma carnavalizada, isto é, às avessas, principalmente quando termina o romance negando a imortalidade da relação entre o narrador e Annabel Lee, dizendo que a arte é a única forma de se manter unido a Lolita:

I am thinking of aurochs and angels, the secret of durable pigments, prophetic sonnets, the refuge of art. And this is the only immortality you and I may share, my Lolita. (NABOKOV, 1999, p. 224)

\title{
Considerações finais
}




\begin{tabular}{|l|l|l|l|l|l|l|}
\hline InterteXto & Uberaba & UFTM & $\begin{array}{l}\text { v. 2 } \\
\text { n. } 2\end{array}$ & p. 20-38 & 2009 - jul. / dez. & ISSN 1981-0601 \\
\hline
\end{tabular}

Analisando o poema Annabel Lee, pudemos perceber que as escolhas feitas na produção do texto, ainda que possam ter sido empíricas, refletem os efeitos visados.

Em análises do poema, além do percurso gerativo de sentido, trata-se da forma como foram construídas as estrofes, com várias repetições gerando no texto o efeito de lamentação por parte do eu ou, também, um efeito de linguagem infantil mesclada à linguagem adulta. Diz-se ainda que a afirmação de que os anjos mataram Annabel é infantil, o que pode significar que desde esse acontecimento traumático, esse eu, que era criança, não cresceu.

Já dentro do percurso, a escolha de pessoas, espaços e tempos enuncivos contrapostos à de pessoas, espaços e tempos enunciativos, conseguiu-se o efeito de que aqueles passaram, e estes permanecem. Dessa forma, Annabel Lee como menina bonita que amava um sujeito, este sujeito, os serafins, o reino à beira-mar e o tempo de sua infância se foram, e o nós que consiste em Annabel Lee e esse sujeito já adulto na posição de narrador que a ama e que sente por ela um apego intenso, o tempo de agora e o espaço sombrio que se caracteriza pelo túmulo onde Annabel está enterrada não deixarão de existir, pois esse narrador enunciador precisa que continuem existindo para garantir sua própria existência semiótica, já que, para ele, só essa junção possui valor.

Lolita, romance escrito mais de cem anos depois de Annabel Lee, é uma obra que retoma a narrativa de Edgar Allan Poe, pois recria uma Annabel Leigh, que também morreu deixando o narrador traumatizado com sua perda. Porém, o romance retoma o poema para negar a imagem romântica feita tanto da menina quanto do narrador: ao criar a Lolita como reencarnação de Annabel, mas sendo totalmente diferente devido à época e tempo em que vive, Nabokov inverte a ordem do mundo de Poe, carnavalizando o amor eterno, idealizado e benevolente que era o tema central daquela obra e transformandoo num amor egoísta e que acaba, por essa razão, sendo uma paixão malevolente. 


\begin{tabular}{|l|l|l|l|l|l|l|}
\hline InterteXto & Uberaba & UFTM & $\begin{array}{l}\text { v. 2 } \\
\text { n. 2 }\end{array}$ & p. 20-38 & 2009 - jul. / dez. & ISSN 1981-0601 \\
\hline
\end{tabular}

\section{Referências}

BARROS, Diana Luz Pessoa de. Teoria Semiótica do Texto. São Paulo: Ática, 2000.

BARROS, Diana Luz Pessoa de; FIORIN, José Luiz (orgs.). Dialogismo, polifonia, intertextualidade. São Paulo: Editora da Universidade de São Paulo, 2003.

EDGAR ALLAN POE SOCIETY OF BALTIMORE. Annabel Lee. Disponível em: http://www.eapoe.org/papers/misc1827/nyt49100.htm\#Annabel_Lee. Acesso em: 27/08/2008.

GREIMAS, A.J.; FONTANILLE, J. Semiótica das Paixões: Dos estados de coisas aos estados de alma. São Paulo: Ática, 1993.

NABOKOV, Vladimir. Lolita. Livro eletrônico disponível em:

http://www.scribd.com/doc/5559866/lolita-vladimir-nabokov. Acesso em:

20/09/2008.

WIKIPEDIA. Annabel Lee. Disponível em:

http://en.wikipedia.org/wiki/Annabel_Lee. Acesso em: 27/08/2008. 